\title{
Plants of the future
}

\author{
Literature is full of descriptions of future utopias and dystopias, but tomorrow's tomorrows are too important to be \\ left to fiction to consider. What qualities will be needed in plants in the coming decades?
}

T he future is not a trivial topic, and yet it is hard to approach unemotionally, and even harder to treat scientifically. Faced with the conflicting predictions of near-magical advances leading to increased gratification and pleasure, and potential problems that could easily lead to disasters, most people try not to think of the future at all. However, the pessimists' problems and the optimists' opportunities are both real, and we must consider both sides rationally so that practical steps can be taken to avert the one and realise the other.

In June 2019, a group of plant scientists assembled in New York to do just that: to brainstorm what plants in the future will look like, what they will need to be capable of and what routes can be followed to bring them into existence. This Nature Conference ("Plants of the Future"), was co-organized with Nature Genetics and our institutional partner, New York University. To facilitate cross-channel discussions, we designed the two-day conference to cover a broad range of topics, including synthetic and systems biology, plant-microorganism interactions, epigenetics, development, environmental stresses and future agriculture.

The United Nations Sustainable Development Goals (SDGs) very clearly set out the challenges to be faced within a relatively small number of years with regards to food supply, energy, medicine, environmental sustainability and much else. Plant sciences can (and will) make tremendous contributions in meeting these needs. We want staple food crops to be high-yield, stress-tolerant, superior-quality and have minimal environmental cost. At the same time, minor crops should increase agricultural diversity and human nutrition. We want energy crops with efficient energy conversion. And all resilient to climate variability. We want more diversity of ornamental plants, more chemicals from medicinal plants, more carbon fixation by natural ecosystems. A lot is riding on plants' shoulders.

The first problem discussed at the Nature Conference was the overuse of synthetic fertilizers. If all major crops could fix nitrogen as the legumes do (by establishing rhizobial symbiosis), we can reduce fertilizer application and worry less about the pollution caused by fertilization.
But is it feasible to establish beneficial association between non-legume plants and the nitrogen-fixing bacteria? This is a very old question, but with new gene-editing tools and a better understanding of what controls the interactions between plants and beneficial microorganisms, we are very close to giving a definitive and affirmative answer.

Alternatively, plants may be made nitrogen-fixing by transgenic approaches, although the success to date is limited. Two hopeful directions are engineering nitrogen fixation into plant-associated bacteria, and screening specific small molecules to enhance the nitrogen fixation of freeliving soil microorganisms. The next steps will be examination of the effects of these approaches in farming practices.

\section{"The future is bright and \\ beautiful. Love it! seek to reach it! work for it! bring it nearer to men! Transfer from it into the present whatever you may be able to transfer" - Nikolay Gavrilovich Chernyshevsky"}

Plant-microorganism interactions also cause diseases leading to great losses of global productivity. Under the context of climate change, Sheng Yang He from Howard Hughes Medical Institute and Michigan State University discussed the effects of humidity and temperature on plant pathogen infection, encouraging the adoption of a multi-dimensional view of 'disease-environment-microbiome' interactions when establishing sustainable agroecosystems.

Given that photosynthesis is the central process of primary production of biomass on the globe, it is no surprise that the question of optimizing photosynthetic efficiency and carbon fixation was much discussed at the New York meeting. Krishna Niyogi, from Howard Huges Medical Institute and University of California, Berkeley, proposed modifying the processes of non-photochemical quenching for the improvement of photosynthetic efficiency.
Another speaker, Moritez Meyer from Martin Jonikas's Group in Princeton University, encouraged plant researchers to borrow wisdom from a green alga, and apply the knowledge gained from the algal $\mathrm{CO}_{2}$-concentrating mechanism to enhance carbon fixation and crop productivity.

We cannot list every direction considered in the meeting, as the Nature Conference accommodated a very broad discussion of the multifaceted challenges being tackled by the plant community; several speakers considered increasing abiotic stress tolerance for example. We will however, continue to showcase the landscape of approaches being used to understand and generate desirable plant traits in the future, so that we can extend the interdisciplinary discussions started at the conference and expand them on a broader scale.

A short, compact meeting can never do more than highlight the problems and opportunities facing a field. The topics specifically discussed need augmenting with additional directions and a larger community of scientists, with different perspectives, to join the conversation. At least one consensus is that, in this era of rapidly advancing technologies, we are exposed to unprecedented opportunities to address problems, both old and new, of food security and environmental sustainability.

Working together on these problems is not only for the current generation. The question of what kind of future our children will inherit must always be near the forefront of our minds. At the very least we can try to intepret the consequences of our everyday activities and the footprint they will leave on our future world. The recent advances towards a sustainable future of plants is a cause for optimism; that we are making efforts to build a better place for our descendants, a planet where they may enjoy growing up without worrying about starvation and pollution. There are, and always will be, new challenges, and the plant community alone does not have all the solutions. But, like our ancestors did time and time again, we must find innovative ways to progress.

Published online: 8 August 2019 https://doi.org/10.1038/s41477-019-0506-9 